\title{
基于灰度能量差异性的红外与可见光图像融合
}

\author{
赵立昌 ${ }^{1}$, 张宝辉 ${ }^{2}$, 吴 杰 $^{2}$, 吴旭东 ${ }^{2}$, 吉 莉 ${ }^{2}$ \\ (1. 南京理工大学 电子工程与光电技术学院, 江苏 南京 210094;
}

2. 昆明物理研究所, 云南 昆明 650223)

\begin{abstract}
摘要: 为了获取红外图像中的突出的目标特征, 提取可见光图像中重要的细节信息, 以及解决传统算 法中目标信息不够突出, 细节、纹理缺失严重的问题, 本文提出了一种基于灰度能量差异性的红外与 可见光图像融合方法。首先通过基于灰度能量差异性的显著目标提取算法检测出红外图像中的目标特 征; 然后采用非下采样轮廓波变换（non-subsampled contourlet transform, NSCT) 对红外图像和可见 光图像进行高低频的分解; 将灰度能量差异图作为融合权重对红外图像和可见光图像的低频部分进行 融合，对于高频部分采用加权方差的规则进行融合; 最后对融合后的高频系数和低频系数进行 NSCT 逆变换得到最终的融合图像。本文选取了 3 组经典的红外与可见光图像进行融合实验, 并且通过主观 视觉和客观指标两个方面与其他几种方法作比较。实验结果证明了算法在突出目标信息、提高对比度、 清晰度和保留纹理细节方面十分有效。
\end{abstract}

关键词：图像融合; 灰度能量差异; NSCT; 加权方差

中图分类号：TP391 文献标识码：A 文章编号：1001-8891(2020)08-0775-08

\section{Fusion of Infrared and Visible Images Based on Gray Energy Difference}

\author{
ZHAO Lichang ${ }^{1}$, ZHANG Baohui ${ }^{2}$, WU Jie ${ }^{2}$, WU Xudong ${ }^{2}, \mathrm{JI} \mathrm{Li}^{2}$ \\ (1. School of Electronic and Optical Engineering, Nanjing University of Science and Technology, Nanjing 210094, China;
}

2. Kunming Institute of Physics, Kunming 650223, China)

\begin{abstract}
This paper proposes an infrared and visible image fusion method based on gray energy difference for two purposes: one, to obtain the prominent target features in an infrared image for extracting the important details in the visible image, and two, to solve the problem that the target information in traditional algorithms is not sufficiently prominent and that the details and textures are often missing. In this method, first, the target feature in the infrared image is detected by a target extraction algorithm based on gray energy difference. Second, infrared and visible images are decomposed to high and low frequencies using a non-subsampled contourlet transform (NSCT). Third, the gray energy difference map is used as the fusion weight to fuse the low-frequency parts of the infrared image and the visible image. The high-frequency part is fused by the rule of weighted variance. Finally, the NSCT inverse transform is used to fuse the high-frequency and low-frequency coefficients to obtain the final fused image. In this study, three groups of classical infrared and visible images are selected for fusion experiments and compared with other methods through subjective vision and objective indicators. Experimental results show that the algorithm can effectively highlight target information, improving contrast and sharpness and retaining texture details.
\end{abstract}

Key words: image fusion, gray energy difference, NSCT, weighted variance 


\section{0 引言}

图像融合技术是将多个图像传感器对同一场景 进行图像采集, 利用各个图像传感器的特点, 将它们 所采集到的图像融合成一幅新的图像。融合后的图像 具有更加丰富的信息 ${ }^{[1]}$, 更适合人眼的观察以及计算 机的后续处理。

红外与可见光图像的融合是图像融合领域的重 要组成部分, 红外图像是根据环境中不同物体所发出 的热辐射差异来探测目标, 因此它适合在低照度或者 目标被隐藏的环境下识别目标, 但是其缺点是细节信 息的缺失; 而可见光图像具有很高的分辨率, 拥有丰 富的细节信息, 但是难以探测出在复杂环境条件下的 目标。而红外与可见光图像的融合可以集成这两种类 型图像的优势 ${ }^{[2]}$, 在计算机视觉、安防监控、地震遥 感等领域有着广泛的应用 ${ }^{[3]}$ 。

目前, 多尺度变换的融合方法是应用于红外与 可见光融合的最广泛的方法。常用的基于多尺度分 解的图像融合方法有: 拉普拉斯金字塔融合算法 ${ }^{[4]}$ 、 离散小波变换法 (discrete wavelet transformation, DWT $)^{[5]}$ 、双树复小波变换 (dual tree complex wavelet transform, DTCWT $)^{[6]}$ 、轮廓波变换 (contourlet transform, CT) ${ }^{[7]}$ 、非下采样轮廓波变换 (NSCT) 等等。 拉普拉斯金字塔分解算法不具备方向性; 离散小波变换 法分解的方向有限, 不能很好地表示细节、轮廓等信息; 双树复小波变换可得到 6 个方向上的高频子带信息, 但 它是冗余的, 对于 $n$ 维信号, 将产生 $2^{n}: 1$ 的圥余度; 轮 廓波变换进行了上、下采样操作, 缺少平移不变性, 容 易产生伪吉布斯效应; 非下采样轮廓波变换是对轮廓波 变换的改善, 在轮廓波的基础上添加了平移不变性, 解 决了伪吉布斯效应的产生, 具有多方向、多尺度的特性, 将 NSCT 应用到图像融合中能够更好地提取源图像的 细节特征和方向信息，保留更多源图像的信息。

融合规则对最终融合图像的质量有着至关重要 的作用, 传统的融合规则有加权平均法 ${ }^{[8]}$ 、绝对值取 大法 ${ }^{[9]}$ 、区域能量法 ${ }^{[10]}$ 等, 这些融合规则没有从图像 区域之间的相关性考虑, 最终的融合效果并不是太理 想。而对红外图像进行显著目标提取的融合算法是根 据人类的视觉机制将人眼感兴趣的部分进行提取, 背 景进行抑制, 融合后的图像具有突显目标特征, 保留 背景细节的优势。已有一些文章对此类算法进行研 究, 主要是对红外图像的显著性区域提取算法的研 究, 如基于文献[11]的改进流形排序的显著性区域检 测, 基于文献[12]的改进 FT (frequency-tuned) 算法 的显著性区域检测和基于文献[13]的形态学滤波的显
著性区域检测等。

为了进一步提高目标和背景之间的对比度, 增强 目标信息的辨识率, 同时尽可能完整地保留可见光图 像中的细节纹理, 本文提出了一种基于灰度能量差异 性的红外与可见光融合方法, 首先通过基于灰度能量 差异性的显著目标提取算法突出红外图像中的目标 信息; 然后使用 NSCT 的分解框架对红外图像和可见 光图像进行高低频的分解; 利用计算得出的灰度能量 差异图对红外图像和可见光图像的低频部分进行融 合, 对于高频部分采用加权方差的规则进行融合; 最 后对融合后的高频系数和低频系数进行 NSCT 逆变换 得到最终的融合图像。

\section{1 基于灰度能量差异性的目标提取算法}

基于灰度能量差异性的目标提取算法是一种在 图像中提取人眼感兴趣的区域的显著目标检测算法, 也是一种显著性检测方法, 主要原理是计算某一灰度 能量与其他灰度能量的差异, 通过能量差异值将图像 中的目标区域提取出来。以往的显著性检测方法, 主 要是基于像素点的灰度值之间的关联进行显著区域 的检测, 如文献[14]和文献[15], 而本文的显著区域检 测算法是基于本文定义的像素点之间的灰度能量的 关系, 对于像素点之间的灰度差异具有更高的敏感 性, 更能突出显著目标。对于一幅 8 位的灰度图像, 定义该幅图像的灰度距离矩阵 $\boldsymbol{D}$ 为:

$$
\boldsymbol{D}=\left[d_{0}, d_{1}, d_{2}, \cdots, d_{k} \cdots, d_{255}\right]
$$

式中: $d_{k}=[k-0, k-1, \cdots, k-255]^{\mathrm{T}}$, 表征像素值为 $k$ 时与其他像素值的灰度距离。定义灰度能量差异值 $E_{k}$ 为:

$$
E_{k}=\sum_{i=1}^{256} w(i) \cdot[D(i, k+1)]^{2}
$$

式中:

$$
w=\frac{1}{N}\left[W_{1}, W_{2}, W_{3}, \cdots, W_{i}, \cdots W_{256}\right]
$$

式中: $W_{i}$ 表示各个像素出现的频数; $N$ 表示图像总共 的像素个数。将灰度能量差异值归一化得:

$$
E N_{k}=\frac{E_{k}-E_{\min }}{E_{\max }-E_{\min }}
$$

式中: $E_{\min }$ 为能量差异最小值; $E_{\max }$ 为能量差异最大 值, 以现在的灰度能量差异值取代原来的像素值, 某 区域灰度能量差异值越大, 说明该区域越引人注意, 是我们需要检测的目标, 而其他灰度能量差异值小的 区域则是我们不感兴趣的地方, 因此可以有效地抑制 背景信息, 突出图像的目标特征。使用正弦函数对灰 度能量差异图进一步增强, 如下式: 


$$
\mathrm{EI}=0.5 \times(\sin (\pi \cdot E N-0.5 \pi)+1)
$$

图 1 是由文献[14]、文献[15]和本文算法所得到的 显著区域比较图。图(a)是红外图像, 图(b)是由文献 [14]的算法所得出的显著图, 图(c)是由文献[15]的算 法得到的显著图, 图(d)是由本文算法所得出的显著 图。传统的图像增强算法在提高图像对比度时无法检 测出显著目标, 会对场景中一些其他的物体也进行增 强, 使得红外图像中细节、纹理等高频信息的缺失的 区域无法有效地抑制, 而后续的低频融合规则会以此 作为融合权重, 这会导致融合后的图像细节也会相应 地缺失, 从而使得成像的清晰度较低, 成像质量较差。 而像文献[14]和文献[15]的显著区域检测算法, 虽然避 免了对图像中高频信息缺失部分的增强, 但是也没有 很好地抑制这些区域。经本文算法处理后的红外图像 能够有效抑制背景, 增强图像中的目标信息, 将人眼 感兴趣的目标提取出来。

\section{NSCT 分解框架}

NSCT 利用非下采样金字塔 (non-subsampled pyramid, NSP) 和非下采样方向滤波器组

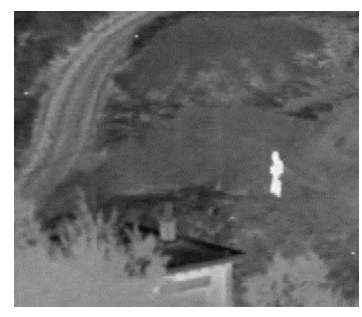

(a) 红外图像

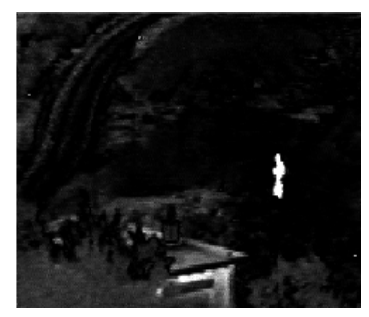

(b) 文献[14]的算法 (a) Infrared image

(b) Paper[14] method （non-subsampled direction filter banks, NSDFB) 实现对 图像的多尺度、多方向的分解。首先通过 NSP 实现多 尺度的分解, 获取高频子带和低频子带, 然后使用 NSDFB 对高频子带进行多方向的分解, 得到高频各 方向子带, 然后对低频子带继续 NSP 分解, 重复上述 过程, 即得到 NSCT 的多层分解, 分解过程如图 2 所 示。NSCT 取消了上、下采样过程, 改为对滤波器直 接采样, 使得变换后得到的每一个子带图像都与源图 像大小相同, 有良好的空域和频域特性, 并且具有平 移不变性 ${ }^{[16]}$ 。

\section{3 本文融合策略}

本文首先通过基于灰度能量差异性的目标检测 算法突出表征红外图像中的目标特征; 然后对红外图 像和可见光图像采用 NSCT 分解得到高频分量和低频 分量; 将基于灰度能量差异性的显著区域提取算法的 结果作为融合权重对红外图像和可见光图像的低频 部分进行融合, 对于高频部分采用加权方差的规则进 行融合; 最后对融合后的高频系数和低频系数进行 NSCT 逆变换得到最终的融合图像。融合框架如图 3 所示。

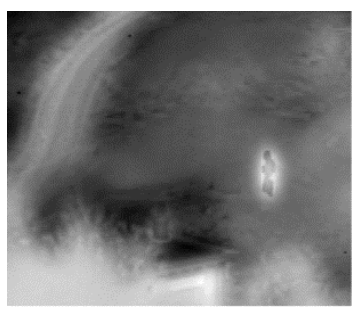

(c) 文献[15]的算法

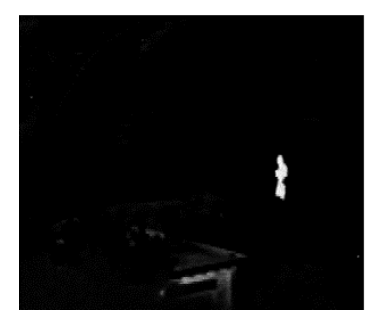

(d) 本文算法 (c) Paper[15] method

(d) Proposed method

图 1 红外图像的显著图比较

Fig.1 Saliency comparison of infrared images

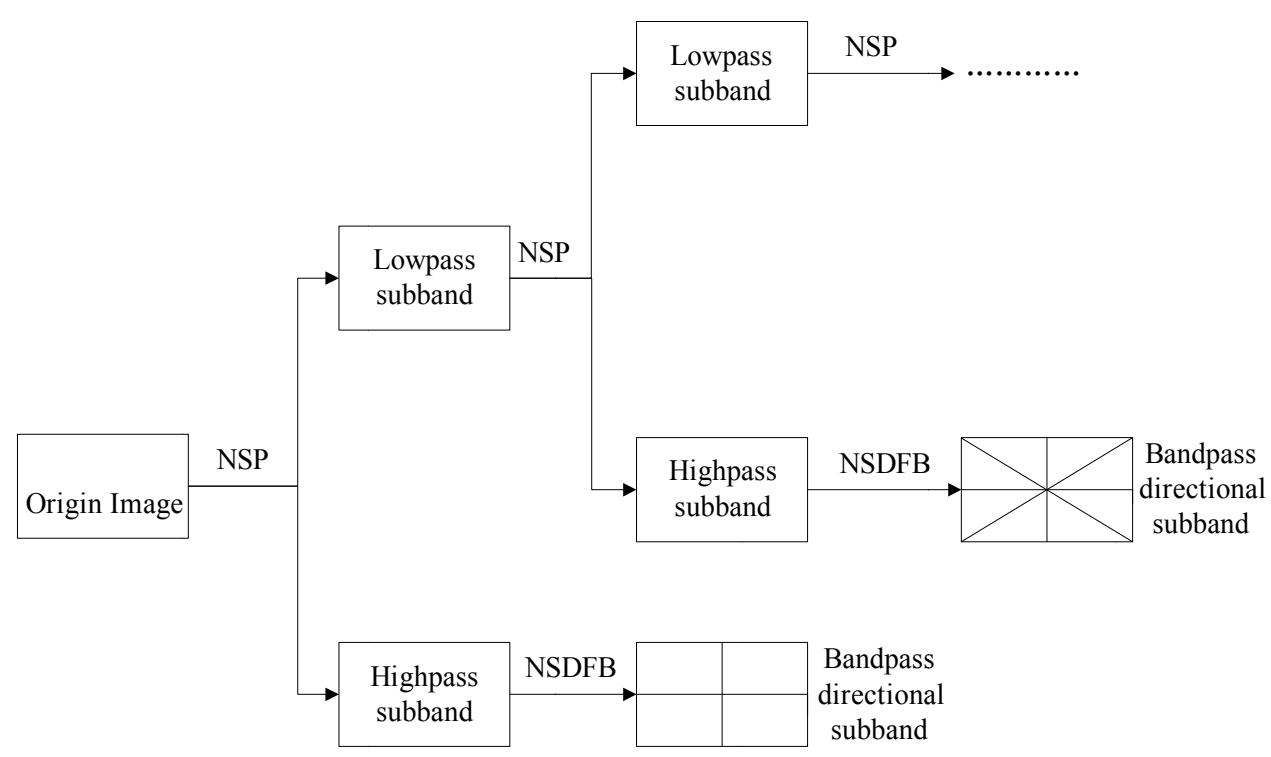

图 $2 \mathrm{NSCT}$ 分解框架示意图

Fig.2 NSCT decomposition framework 


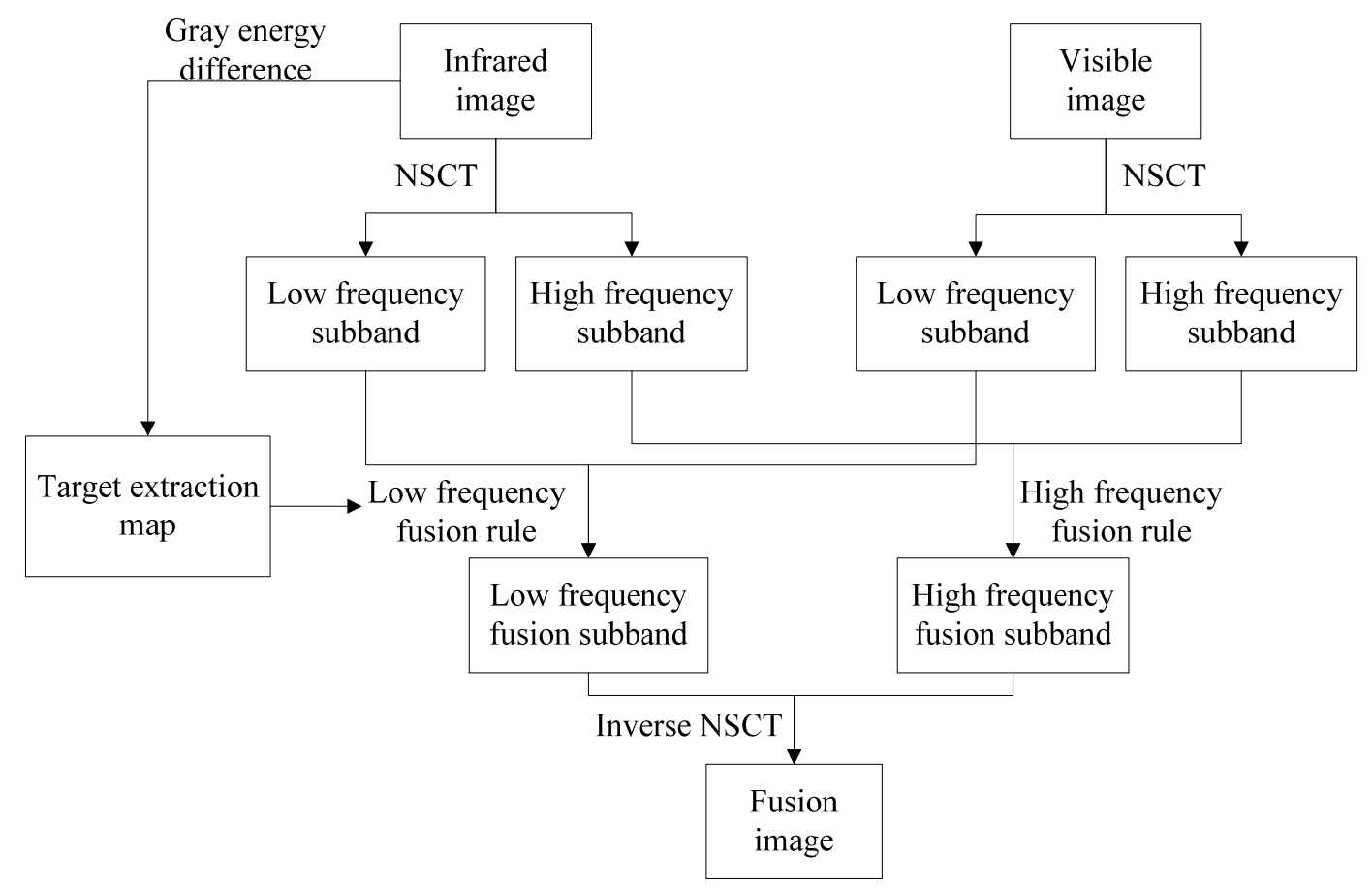

图 3 本文算法的融合框架

\section{1 低频系数融合规则}

由于人眼的视觉系统主要是对图像中像素的对 比度较为敏感, 对像素的亮度不太敏感, 背景等灰度 变化较为缓慢的区域被保留在低频子带中, 而传统的 低频系数融合方法无法清唽地区分出目标轮廓与背 景信息, 全局对比度较低, 本文提出了一种基于灰度 能量差异的方法能够有效地提取红外图像中的目标 区域。设红外图像和可见光图像经 NSCT 后所得到的 低频系数分别为 $C_{\mathrm{L}}{ }^{\mathrm{IR}}(i, j)$ 和 $C_{\mathrm{L}}{ }^{\mathrm{VIS}}(i, j)$, 由本文算法所得 到灰度能量差异图为 $\mathrm{EI}$, 则融合后的低频系数为:

$$
C_{\mathrm{L}}^{\mathrm{F}}(i, j)=w_{\mathrm{L}}^{\mathrm{IR}}(i, j) C_{\mathrm{L}}^{\mathrm{IR}}(i, j)+w_{\mathrm{L}}^{\mathrm{VIS}}(i, j) C_{\mathrm{L}}^{\mathrm{VIS}}(i, j)
$$

其中融合权重 $w_{\mathrm{L}}{ }^{\mathrm{IR}}$ 和 $w_{\mathrm{L}}^{\mathrm{VIS}}$ 的值由下式可得:

$$
\begin{array}{r}
w_{\mathrm{L}}^{\mathrm{IR}}(i, j)=\mathrm{EI}(i, j) \\
w_{\mathrm{L}}^{\mathrm{VIS}}(i, j)=1-\mathrm{EI}(i, j)
\end{array}
$$

\section{2 高频系数融合规则}

经 NSCT 分解得到的高频子带能够表征图像的边 缘、纹理等细节信息。本文的高频系数融合规则是基 于方差制定。方差的大小可以表示为该区域包含细节 信息的多少, 方差越大, 说明该点与周围区域的灰度 值差距越大, 在图像画面中表现的就是纹理等高频信 息越丰富, 本文所设定的方差分辨阈值主要是用来比 较红外图像与可见光图像高频部分方差的大小, 本文 所取的阈值为 0.24 。由数学关系可知, 两者所占的权 重之和为定值 1 , 则权重之积最大为 0.25 , 此时表示
红外图像与可见光图像的权重各占 50\%, 说明两幅图 像有着近乎相同细节信息。当一方所占权重 $\geqslant 40 \%$ 并 且 $\leqslant 50 \%$, 即权重之积大于等于 0.24 , 可以认为两者 方差之差较小, 拥有相似的细节信息, 两幅图像的高 频部分对最终的融合图像的高频部分均有较大贡献, 此时的融合图像的高频信息使用加权融合的方法; 当 一方所占权重小于 $40 \%$, 即权重之积小于 0.24 , 说明 两幅图像的方差之差较大, 一方含有较多的细节信 息, 而另一方的细节信息较少, 最终的融合图像的高 频部分主要来自于方差大的一方, 此时高频部分采用 方差取大的融合方法。由于高频部分的融合规则是基 于方差制定的, 所以对于不同场景都能取得较好的融 合效果。

设 $T$ 为分辨两幅图局部方差差距的判断依据, $T$ 的表达式为:

$$
T(i, j)=w_{\mathrm{H}, k}^{\mathrm{IR}}(i, j) \cdot w_{\mathrm{H}, k}^{\mathrm{VIS}}(i, j)
$$

式中: $w_{\mathrm{H}, k}^{\mathrm{IR}}(i, j)$ 表示在红外图像 $k$ 方向上的高频子带 在像素点 $(i, j)$ 处所占的权重; $w_{\mathrm{H}, k}^{\mathrm{VIS}}(i, j)$ 则表示可见光 图像所占的比重。权重表达式可由下式求得:

$$
\begin{aligned}
& w_{\mathrm{H}, k}^{\mathrm{IR}}(i, j)=\frac{S_{\mathrm{H}, k}^{\mathrm{IR}}(i, j)}{S_{\mathrm{H}, k}^{\mathrm{IR}}(i, j)+S_{\mathrm{H}, k}^{\mathrm{VIS}}(i, j)} \\
& w_{\mathrm{H}, k}^{\mathrm{VIS}}(i, j)=\frac{S_{\mathrm{H}, k}^{\mathrm{VIS}}(i, j)}{S_{\mathrm{H}, k}^{\mathrm{I}}(i, j)+S_{\mathrm{H}, k}^{\mathrm{VIS}}(i, j)}
\end{aligned}
$$

式中: $S_{\mathrm{H}, k}^{\mathrm{IR}}(i, j)$ 表征红外图像 $k$ 方向上的高频子带在 像素点 $(i, j)$ 处的方差; $S^{\mathrm{VIS}}{ }_{\mathrm{H}, k}(i, j)$ 表征可见光图像的方 差, 其具体表达式如下: 


$$
\begin{aligned}
& S_{\mathrm{H}, k}^{\mathrm{IR}}(i, j)= \\
& \frac{1}{M \times N} \sum_{m=-(M-1) / 2}^{(M-1) / 2} \sum_{n=-(N-1) / 2}^{(N-1) / 2}\left[C_{\mathrm{H}, k}^{\mathrm{IR}}(i+m, j+n)-\mu\right]^{2} \\
& S_{\mathrm{H}, k}^{\mathrm{VIS}}(i, j)= \\
& \frac{1}{M \times N} \sum_{m=-(M-1) / 2}^{(M-1) / 2} \sum_{n=-(N-1) / 2}^{(N-1) / 2}\left[C_{\mathrm{H}, k}^{\mathrm{VIS}}(i+m, j+n)-\mu\right]^{2}
\end{aligned}
$$

式中: $C_{\mathrm{H}, k}^{\mathrm{IR}}(i+m, j+n)$ 表示红外图像 $k$ 方向上的高频 子带在该点的灰度值; $C_{\mathrm{H}, k}^{\mathrm{VIS}}(i+m, j+n)$ 表示可见光图 像 $k$ 方向上的高频子带在该点的灰度值; $\mu$ 为区域内 的均值; $M$ 和 $N$ 表示区域窗口大小。高频融合规则可 由下式表示:

当 $T<0.24$ 时:

$$
C_{\mathrm{H}, k}^{\mathrm{F}}(i, j)=\left\{\begin{array}{l}
C_{\mathrm{H}, k}^{\mathrm{IR}}(i, j), S_{\mathrm{H}, k}^{\mathrm{IR}}(i, j)>S_{\mathrm{H}, k}^{\mathrm{VIS}}(i, j) \\
C_{\mathrm{H}, k}^{\mathrm{VIS}}(i, j), S_{\mathrm{H}, k}^{\mathrm{IR}}(i, j)<S_{\mathrm{H}, k}^{\mathrm{VIS}}(i, j)
\end{array}\right.
$$

当 $T \geqslant 0.24$ 时:

$$
\begin{aligned}
& C_{\mathrm{H}, k}^{\mathrm{F}}(i, j)=w_{\mathrm{H}, k}^{\mathrm{IR}}(i, j) C_{\mathrm{H}, k}^{\mathrm{IR}}(i, j)+ \\
& w_{\mathrm{H}, k}^{\mathrm{VIS}}(i, j) C_{\mathrm{H}, k}^{\mathrm{VIS}}(i, j)
\end{aligned}
$$

式中: $C_{\mathrm{H}, k}^{\mathrm{F}}$ 表征两幅图像在 $k$ 方向上的高频子带融合 后的高频系数。

\section{4 实验结果与分析}

为验证本文算法的有效性, 选取 3 组典型的已配 准的红外与可见光图像进行融合实验, 并将本文算法

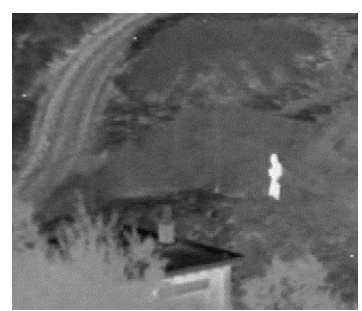

(a) 红外图像

(a) Infrared image

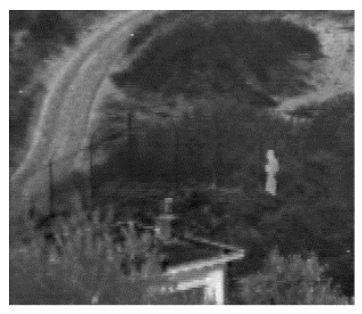

(e) NSCT 方法

(e) NSCT method

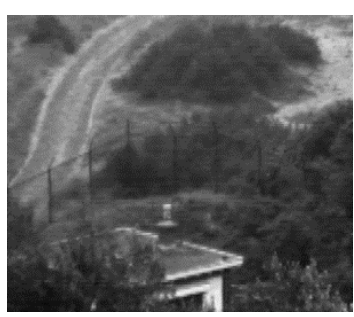

(b) 可见光图像

(b) Visble image

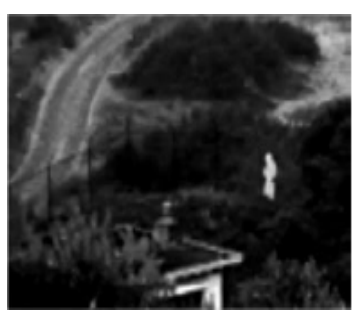

(f) 文献[17]算法

(f) Paper[17] method

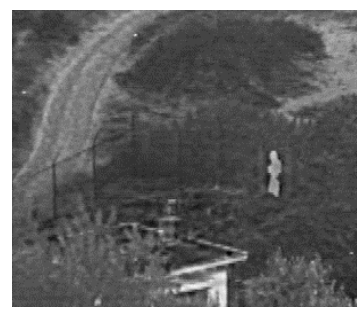

(c) DWT 方法

(c) DWT method

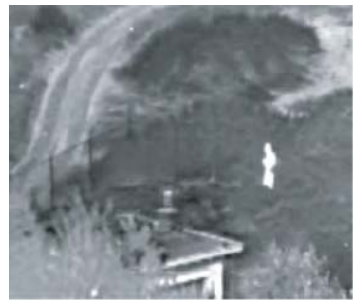

(g) 文献[18]算法

(g) Paper[18] method

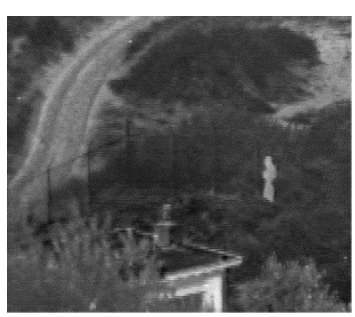

(d) DTCWT 方法

(d) DTCWT method

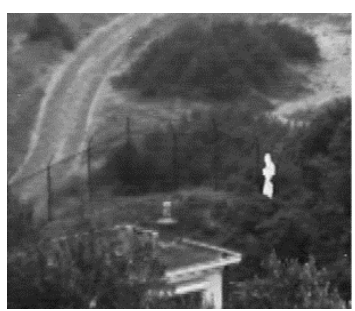

(h) 本文算法

(h) Proposed method

图 4 Uncamp 源图像融合结果

Fig.4 Fusion results of Uncamp source images 
图 6 为 Kayak 源图像融合结果。可以看出图(c)、 (d)、(e)对比度较低, 视觉效果较差; 图(f)有明显的阴 影; 图 $(\mathrm{g})$ 保留的背景信息较为完整, 但是整体亮度偏 高, 不符合人眼观察; 图(h)是由本文算法计算出的融 合图像, 清晰度、对比度适宜, 无伪影, 亮度适中, 视觉观感最佳。

根据以上 3 组图片, 通过主观视觉比较可以看出 本文算法在图像融合的成像质量、视觉效果上有着较 大的优势, 也可以通过客观参数指标对这几种算法进

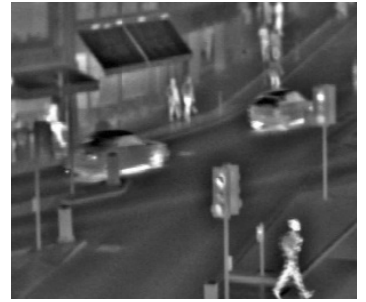

(a) 红外图像

(a) Infrared image

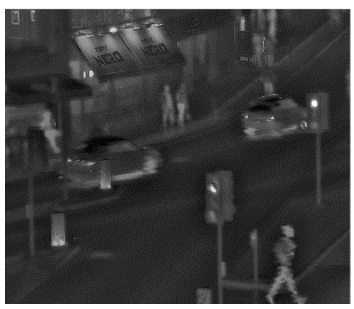

(e) NSCT 方法

(e) NSCT method

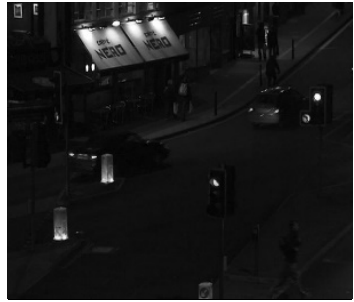

(b) 可见光图像

(b) Visble image

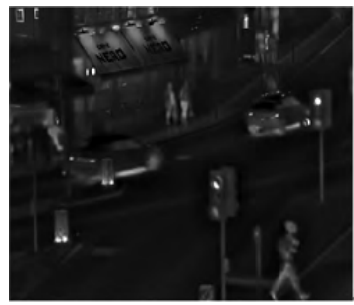

(f) 文献[17]算法

(f) Paper[17] method

行比较, 如表 1 所示。

从表 1 中可以看出本文算法在信息熵 （information entropy, IE)、平均梯度 (average gradient, AD）、标准差（standard deviation, STD）、空间频率 （spatial frequency, SF）这 4 个指标中均位于前列, 说 明由本文算法处理所得的融合图像从源图像中获得 的信息量最多、拥有更丰富的细节特征、对比度和边 缘保持能力好、背景与目标可清晰分辨, 与主观视觉 所得出的结论相符。

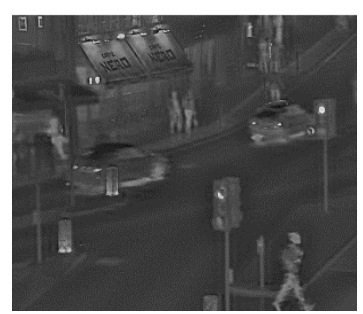

(c) DWT 方法

(c) DWT method

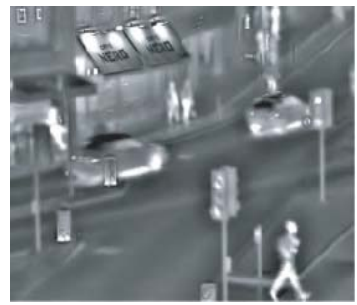

(g) 文献[18]算法

(g) Paper[18] method

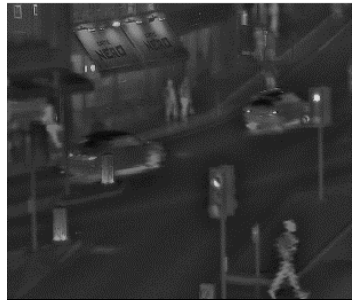

(d) DTCWT 方法

(d) DTCWT method

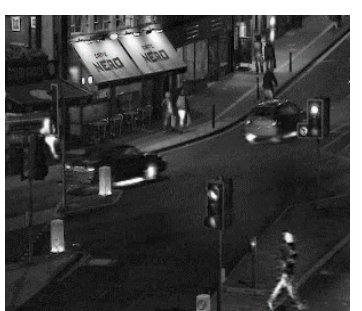

(h) 本文算法

(h) Proposed method

图 5 Quad 源图像融合结果

Fig.5 Fusion results of Quad source images

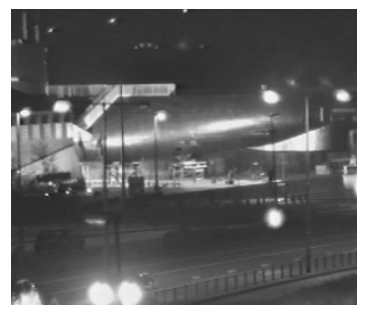

(a) 红外图像

(a) Infrared image

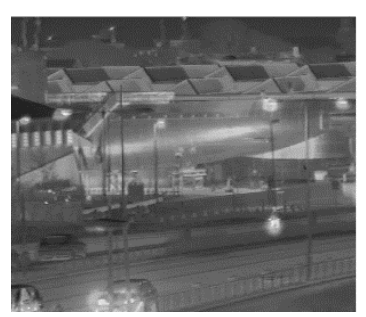

(e) NSCT 方法

(e) NSCT method

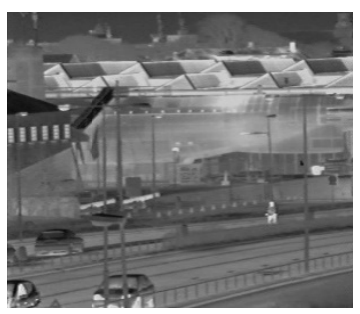

(b) 可见光图像

(b) Visble image

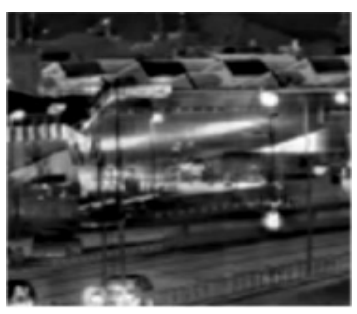

(f) 文献[17]算法

(f) Paper[17] method

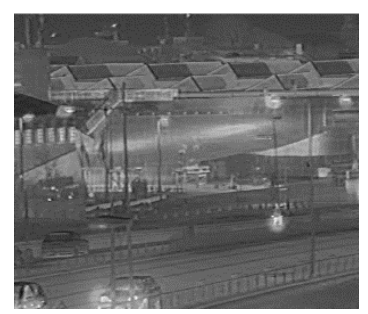

(c) DWT 方法

(c) DWT method

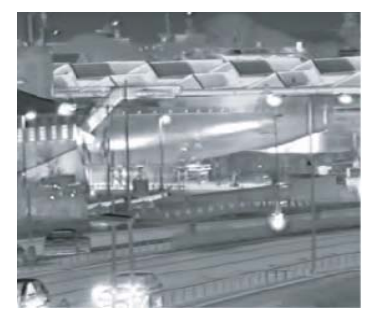

(g) 文献[18]算法

(g) Paper[18] method

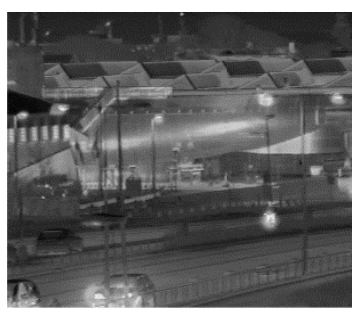

(d) DTCWT 方法

(d) DTCWT method

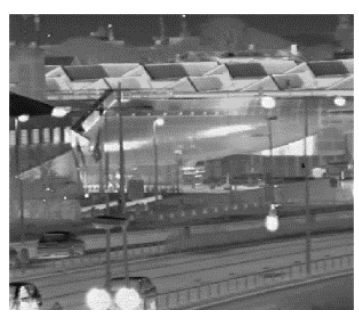

(h) 本文算法

(h) Proposed method

图 6 Kayak 源图像融合结果

Fig.6 Fusion results of Kayak source images 


\begin{tabular}{|c|c|c|c|c|c|c|c|}
\hline Image & $\begin{array}{l}\text { Objective } \\
\text { indicators }\end{array}$ & DWT & DTCWT & NSCT & Paper[15] & Paper[16] & $\begin{array}{c}\text { Proposed } \\
\text { method }\end{array}$ \\
\hline \multirow{4}{*}{ Uncamp } & STD & 23.0572 & 22.9816 & 22.9621 & 30.3326 & 37.4128 & 37.9257 \\
\hline & IE & 6.2928 & 6.2881 & 6.2824 & 6.5456 & 6.8546 & 7.0483 \\
\hline & $\mathrm{AG}$ & 5.1213 & 4.9704 & 4.8633 & 5.3689 & 5.9631 & 6.0814 \\
\hline & $\mathrm{SF}$ & 10.5506 & 10.2025 & 9.9327 & 10.1569 & 11.0176 & 11.4267 \\
\hline \multirow{4}{*}{ Quad } & STD & 21.2288 & 21.219 & 21.1939 & 32.0256 & 38.4109 & 39.151 \\
\hline & IE & 5.9358 & 5.9347 & 5.9332 & 6.1254 & 6.3245 & 6.6146 \\
\hline & $\mathrm{AG}$ & 3.2197 & 3.1769 & 3.1342 & 3.9869 & 4.1963 & 6.7246 \\
\hline & $\mathrm{SF}$ & 10.2286 & 10.1542 & 10.0335 & 11.5694 & 13.2459 & 16.4883 \\
\hline \multirow{4}{*}{ Kayak } & STD & 30.7832 & 30.8162 & 30.7468 & 35.5632 & 41.2459 & 39.1572 \\
\hline & IE & 6.8183 & 6.8192 & 6.8156 & 6.8896 & 6.8812 & 7.1731 \\
\hline & $\mathrm{AG}$ & 4.7269 & 4.6205 & 4.5955 & 5.1225 & 6.3129 & 6.4752 \\
\hline & $\mathrm{SF}$ & 9.2457 & 9.0361 & 8.922 & 11.0517 & 12.1509 & 11.9222 \\
\hline
\end{tabular}

\section{5 结论}

本文提出了一种基于灰度能量差异性的红外与 可见光图像融合方法, 通过对红外图像中目标信息的 增强提取, 融合后的目标特征更加显著, 并且极大地 保留了可见光图像中的细节, 有效地解决了传统融合 算法时出现的目标信息不够突出, 细节、纹理模糊等 缺点, 并且利用 3 组经典的红外与可见光图像进行融 合实验, 实验结果表明本文算法较其他算法清晰度更 高, 对比度更适宜, 更适合人眼观察, STD、IE、AG、 $\mathrm{SF}$ 等客观评价指标所得出的结论也与主观视觉感受 一致, 说明本文融合算法是一种行之有效的红外与可 见光图像融合算法。

\section{参考文献：}

[1] TIAN Zhuxiang, LI Yan, GAO Rongrong. A fusion algorithm for infrared and visible images based on adaptive dual-channel unit-linking PCNN in NSCT domain[J]. Infrared Physics and Technology, 2015, 69: 53-61.

[2] 徐冠雷, 王孝通, 徐晓刚, 等. 基于限邻域经验模式分解的多波段图 像融合 [J]. 红外与毫米波学报, 2006(3): 225-228.

XU Guanlei, WANG Xiaotong, XU Xiaogang, et al. Multi-band image fusion algorithm based on neighborhood limited empirical mode decomposition[J]. Journal of Infrared and Millimeter Waves, 2006(3): $225-228$.

[3] ZHAO J, GAO X, CHEN Y, et al. Multi-window visual saliency extraction for fusion of visible and infrared images[J]. Infrared Physics \& Technology, 2016, 76: 295-302.

[4] 陈浩, 王延杰. 基于拉普拉斯金字塔变换的图像融合算法研究[J]. 激
光与红外, 2009, 39(4): 439-442.

CHEN Hao, WANG Yanjie. Research on image fusion algorithm based on Laplacian pyramid transform[J]. Laser \& Infrared, 2009, 39(4): $439-442$.

[5] TIAN Pu, GUO Giangni. Contrast-based image fusion using the discrete wavelet transform[J]. Optical Engineering, 2000, 39(8): 2075.

[6] 朱攀, 刘泽阳, 黄战华. 基于 DTCWT 和稀疏表示的红外偏振与光强 图像融合 $[J]$. 光子学报, 2017, 46(12): 213-221.

ZHU Pan, LIU Zeyang, HUANG Zhanhua. Infrared polarization and intensity image fusion based on dual-tree complex wavelet transform and sparse representation[J]. Acta Photonica Sinica, 2017, 46(12): 213-221.

[7] Do Minh N, Vetterli Martin. The contourlet transform: an efficient directional multiresolution image representation[J]. IEEE Transactions on Image Processing: a Publication of the IEEE Signal Processing Society, 2005, 14(12): 2091-2106.

[8] LI Huafeng, QIU Hongmei, YU Zhengtao, et al. Infrared and visible image fusion scheme based on NSCT and low-level visual features[J]. Infrared Physics and Technology, 2016, 76: 174-184.

[9] 王珺, 彭进业, 何贵青, 等. 基于非下采样 Contourlet 变换和稀疏表示 的红外与可见光图像融合方法 [J]. 兵工学报, 2013, 34(7): 815-820. WANG Jun, PENG Jinye, HE Guiqing, et al. Fusion method for visible and infrared images based on non-subsampled contourlet transform and sparse representation[J]. Acta Armamentarii, 2013, 34(7): 815-820.

[10] 刘先红，陈志斌. 基于多尺度方向引导滤波和卷积稀疏表示的红外 与可见光图像融合 $[\mathrm{J}]$. 光学学报, 2017, 37(11): 111-120.

LIU Xianhong, CHEN Zhibin. Fusion of infrared and visible images based on multi-scale directional guided filter and convolution sparse 
representation[J]. Acta Optica Sinica, 2017, 37(11): 111-120

[11] 易翔, 王炳健. 视觉显著性指导的红外与可见光图像融合算法 $[\mathrm{J}]$. 西 安电子科技大学学报, 2019, 46(1): 27-32, 38 .

YI Xiang, WANG Bingjian. Fusion of infrared and visual images guided by visual saliency[J]. Journal of XIDIAN University, 2019, 46(1): 27-32, 38.

[12] 林子慧, 魏宇星, 张建林, 等. 基于显著性图的红外与可见光图像融 合 $[\mathrm{J}]$. 红外技术, 2019, 41(7): 640-645.

LIN Zihui, WEI Yuxing, ZHANG Jianlin, et al. Image fusion of infrared and visible images based on saliency map[J]. Infrared Technology, 2019, 41(7): 640-645.

[13] 张宝辉, 闵超波, 窦亮, 等. 目标增强的红外与微光图像融合算法[J]. 红外与激光工程, 2014, 43(7): 2349-2353.

ZHANG Baohui, MIN Chaobo, DOU Liang, et al. Fusion algorithm of target enhancing infrared and low-level-light image[J]. Infrared and Laser Engineering, 2014, 43(7): 2349-2353.

[14] ZHAO Jufeng, ZHOU Qiang. Fusion of visible and infrared images using saliency analysis and detail preserving based image decomposition[J]. Infrared Physics \& Technology, 2013, 56(2013):
93-99.

[15] Achanta R, Hemami S, Estrada F, et al. Frequency-tuned salient region detection[C]//Computer Vision and Pattern Recognition of IEEE, 2009: 1597-1604.

[16] 陈震, 杨小平, 张聪炫, 等. 基于补偿机制的 NSCT 域红外与可见光 图像融合[J]. 仪器仪表学报, 2016, 37(4): 860-870.

CHEN Zhen, YANG Xiaoping, ZHANG Congxuan, et al. Infrared and visible image fusion based on the compensation mechanism[J]. Chinese Journal of Scientific Instrument, 2016, 37(4): 860-870.

[17] 王焕清. 结合 NSCT 和邻域特性的红外与可见光图像融合 [J]. 信息通 信, 2018(4): 17-20.

WANG Huanqing. Image fusion visible and infrared image based on NSCT and neighborhood features[J]. Information and Communication, 2018(4): 17-20.

[18] 刘先红, 陈志斌, 秦梦泽. 结合引导滤波和卷积稀疏表示的红外与可 见光图像融合 $[\mathrm{J}]$. 光学精密工程, 2018, 26(5): 1242-1253.

LIU Xianhong, CHEN Zhibin, QIN Mengze. Infrared and visible image fusion using guided filter and convolutional sparse representation[J]. Optics and Precision Engineering, 2018, 26(5): 1242-1253. 